\title{
Bioinformatic analysis and identification of potential hallmarks in poorly differentiated thyroid cancer
}

\section{Xin Chen}

The First Affiliated Hospital of Zhengzhou University

\section{Runsheng Ma}

The First Affiliated Hospital of Zhengzhou University

\section{Hongqiang Li}

The First Affiliated Hospital of Zhengzhou University

\section{Yifeng Tang}

The First Affiliated Hospital of zhengzhou University

\section{Detao Yin ( $\nabla$ detaoyin@zzu.edu.cn )}

The First Affiliated Hospital of Zhengzhou University https://orcid.org/0000-0002-4349-6857

\section{Primary research}

Keywords: poorly differentiated thyroid cancer, malignant transformation, bioinformatic analysis, Hub genes, cell adhesion, EGF

Posted Date: January 29th, 2021

DOI: https://doi.org/10.21203/rs.3.rs-153940/v1

License: (c) (i) This work is licensed under a Creative Commons Attribution 4.0 International License. Read Full License 


\section{Abstract}

Purpose: The accumulation of malignant tumor gene mutations makes differentiated thyroid cancer (DTC) gradually dedifferentiated to poorly differentiated thyroid cancer (PDTC) or anaplastic thyroid cancer (ATC). This study analyzed the gene expression profile in the process of dedifferentiation of thyroid cancer, aiming to explore the molecular mechanism of PDTC and ATC.

Methods: Eight series from GEO database are collected for differentially expressed genes (DEGs) of DTC, PDTC and ATC. Then, GO analysis, KEGG pathway analysis, and functional path enrichment analyses are performed by MATESCAPE. Hub-genes are identified by using the method of MNC in protein interaction networks. Moreover, the expression of hub-genes and hub-gene related survival in thyroid cancers are analyzed by GEPIA2. Finally, the functional path enrichment pathways of PDTC are performed by GSEA Java.

Results: There are obvious differences among DEGs of DTC, PDTC, and ATC, and 17 cross gene of DEGs were found. The DEGs in PDTC were mainly concentrated in regulation of cytoskeleton organization, cell division, positive regulation of kinase activity. While the DEGs in ATC were mainly in extracellular matrix organization, extracellular structure organization. Furthermore, 6 hub-genes were obtained in the development of PDTC by using Cytoscape: EGF, CCND1, DEPDC1, ANLN, HGF, BCL2L1. The high expression levels of the genes of EGF, DEPDC1, ANLN, HGF were associated with the poor survival of thyroid cancer. While the high expression levels of CCND1 and BCL2L1 were beneficial to the survival of patients with thyroid tumor. Finally, GSEA revealed that two gene sets are significantly enriched, including KEGG_THYROID_CANCER and KEGG_GLIOMA. And CCND1 and EGF have a core gene position in KEGG_GLIOMA.

Conclusions: The molecular function (MF), biological processes (BP) and KEGG pathways have changed during the process of malignant transformation of thyroid cancer. Especially, the activation of EGF-EGFR pathway promotes the malignant transformation of thyroid tumor. Furthermore, six hub-genes, especially CCND1 and EGF, could become potential biomarkers of PDTC. This study can serve as a reference to understand the malignant transformation of thyroid cancer.

\section{Introduction}

In recent years, the incidence of patients with thyroid tumors has shown a continuous and obvious increasing trend worldwide[1-3]. According to "Cancer Statistics, 2018", the global incidence of thyroid tumor patients has risen to the fifth place among female systemic malignancies[4]. Based on the degree of differentiation, thyroid cancer could be classified as differentiated thyroid carcinoma (DTC), poorly differentiated thyroid carcinoma (PDTC) and anaplastic thyroid cancer (ATC). Basic research has found that changes of MAPK signaling pathway and PI3K-AKT signaling pathway are mainly involved in the formation of papillary thyroid cancer and follicular thyroid cancer, respectively. As the grade of thyroid tumor improves, the signal molecules of the two pathways become activated more frequently[5-7]. And when genes in the MAPK signal pathway are mutated, it will drive thyroid cells to papillary thyroid cancer; while the PI3K-AKT signal pathway is activated, it will drive the occurrence of follicular thyroid cancer[5].

Several rare types of BRAF mutations found in papillary thyroid cancer mainly affect the nucleotides around codon 600 and activate BRAF kinase. The activated BRAF kinase promotes the high expression of its downstream signal transduction pathways of MAPK, leading to excessive proliferation of thyroid cancer cells[8,9]. Studies have found that BRAF V600E is closely related to the malignant pathological manifestations of papillary thyroid cancer, including aggressive pathological features, increased recurrence, and loss of radioactive iodine affinity [10, 11]. Animal experiments have proved that the expression of the mutant BRAF-V600E in transgenic rats can promote goiter enlargement and produce invasive growth of papillary thyroid carcinoma [12]. In addition to the BRAF-V600E mutation, 
RAS mutations are also common mutations in thyroid cancer, which encodes the protein of p21. When it is mutated and binds to GTP, RAS is activated. There are three hotspots of RAS mutations: HRAS, KRAS and NRAS. Among them, NRAS mutation is the most common RAS mutation in thyroid tumors, mainly involving codons 12 and 61[13]. RAS mutations are a kind of classic dual activator of MAPK and PI3K-AKT signaling pathways, but RAS mutations in thyroid tumors seem to preferentially activate PI3K-AKT signaling pathways. RAS mutations are priority to combine with phosphorylated AKT in thyroid cancer $[12,14]$.Studies have found that with the accumulation of mutated genes and the activation of two pathways, differentiated thyroid cancer can continue to progress to poorly differentiated thyroid cancer (PDTC) and anaplastic thyroid cancer (ATC)[15]. And gene mutations (including TP53, CTNNB1 and ALK mutations) further accelerate the occurrence and development of this process[16-18].

However, poorly differentiated thyroid cancer and anaplastic thyroid cancer are not easy to detect and the results of surgery are dissatisfactory, and many anaplastic thyroid cancer ptietients even lose the opportunity for surgery. The

treatment of ${ }^{131} \mathrm{I}$ is even more unsatisfactory, and the prognosis is significantly poor. According to statistics, the 5 - year survival rate of poorly differentiated thyroid cancer is only $50 \%$, which is far lower than $95 \%$ of differentiated thyroid cancer. And the average survival time of anaplastic thyroid cancer is only $3-6$ months[19, 20]. Early and accurate diagnosis of poorly differentiated thyroid cancer and undifferentiated thyroid cancer is particularly important. But the molecular mechanism of PDTC is not well understood, and there are relatively few studies about hallmarks of PDTC. Recently with the development of microarray and next-generation sequencing technologies, a growing number of microarray and sequencing data have been explored to seek novel biomarkers and therapeutic targets for thyroid cancer[21]. However, small sample sizes in individual studies and different Technological platforms create substantial inter-study variability and difficult statistical analyses. To solve this problem, integrated bioinformatics meta analysis have been utilized in various cancer studies[22]. This research aims to explore the key hallmark genes in the process of dedifferentiation of thyroid cancer. And we will further explore the molecular mechanism of PDTC and ATC, and provide a theoretical basis for its early accurate diagnosis and precise treatment.

\section{Methods}

\section{(1) Identification of DEGs in thyroid cancer}

As shown in Table 1, eight series (including GSE3467, GSE3678, GSE27155, GSE53072, GSE65144, GSE76039, GSE82208, GSE53157) are obtained by searching GEO database with keyword of thyroid cancer, and limited the species as Homo sapiens. The data of mRNA expression are extracted and classfied as DTC, PDTC, ATC, normal thyroid. And they are grouped into: DTC-NTH (Normal thyroid); PDTC-NTH, PDTC-DTC; ATC-NTH, ATC-DTC, ATC-PDTC. The raw expression data are processed and normalized by $\mathrm{R}$ languae with package of "Affy" and "limma". The missing values are calculated by the method of KNN. The inter-batch differences (combat) are removed through R package of "sva". The DEGs expression profile is calculated by Bayesian test with R package of "futile.logger", with parameters of $p$-value $<0.05 \&$ abs ( $\log 2$ fold change difference) $>0.5$. And then the heatmap of DEGs are conducted and clustered by using $R$ package of "gplots". 
Table 1

The information of GSE series in thyroid cancer.

\begin{tabular}{|lllll|}
\hline \multicolumn{5}{|c|}{ Sample } \\
\hline Series & DTC & PDTC & ATC & Normal thyroid \\
\hline GSE3467 & 9 & & & 9 \\
\hline GSE3678 & 7 & & 7 \\
\hline GSE27155 & 64 & & 4 & 4 \\
\hline GSE53072 & & & 5 & 4 \\
\hline GSE65144 & & & 12 & 13 \\
\hline GSE76039 & & 17 & 20 & \\
\hline GSE82208 & 27 & & & \\
\hline GSE53157 & 19 & 5 & & \\
\hline
\end{tabular}

\section{(2) Functional enrichment analysis of DEGs in DTC, PDTC, and ATC}

Metascape (http://metascape.org) provides intuitive visualizations for either pathway enrichment networks or proteinprotein interaction networks. The DEGs of DTC-ALL, PDTC-ALL, ATC-ALL are analyzed for the KEGG Pathway, the GO Biological Processes, biological response and signal pathways database Reactome Pathway Database, classical pathway Canonical Pathways, mammalian protein complex database CORUM, which are conducted in MATESCAPE. The DEGs list of DTC-ALL is equal to that of DCT-NTH. The DEGs list of PDTC-ALL is merged by that of PDTC-NTH and PDTC-DTC. And the DEGs list of ATC-All is merged by ATC-NTH, ATC-DTC, and ATC-PDTC.

\section{(3) Functional enrichment analysis of DEGs in PDTC-NTH and PDTC-DTC}

Further the DEGs of PDTC-NTH and PDTC-DTC are analyzed for the KEGG Pathway, the GO Biological Processes, biological response and signal pathways database Reactome Pathway Database, classical pathway Canonical Pathways, mammalian protein complex database CORUM, which are conducted in MATESCAPE. When multiple gene lists of PDTC-NTH and PDTC-DTC are provided, all lists are merged into one list called "PDTC_ALL".

\section{『4囚Comprehensive co-Expression Network Analysis and Candidate Biomarker in PDTC}

STRING (https://string-db.org/) is an online searching tool for retrieving interacting genes that predicts the qualitycontrolled PPI networks. We used STRING to construct a protein interaction network, and perform the gene coexpression analysis. Cytoscape (https://cytoscape.org/) is an open-source software platform for visualizing complex networks and integrating networks from the attributing data. We visualized the results of the protein interaction network of STRING through Cytoscape. In the present study, the hub genes are calculated by the method of MNC. 


\section{囚5囚Relationship between the Expression of Hub genes and Clinical Features of thyroid cancer}

Gene Expression Profiling Interactive Analysis (GEPIA, http://gepia.cancer-pku.cn/index.html) is an interactive web resource for analyzing cancer transcriptome data, enabling researchers to collect valuable data on genes with interesting information. We performed a survival analysis of hub-genes by using GEPIA.

\section{『6冈اmmunohistochemical Evaluation of hub gene Protein Expression as a Biomarker}

The human protein atlas (https://www.proteinatlas.org/) contains information regarding the expression profiles of human genes both on the mRNA and protein level. The protein level of EGF, CCND1, DEPDC1, ANLN, HGF, BCL2L1 in thyroid cancer and thyroid tissue are searched through the human protein atlas database.

\section{Q7凶GSEA reveal a close relationship between hub genes and tumor transformation}

Gene set enrichment analysis (GSEA) is a computational method that determines whether the members of a gene set are randomly distributed throughout the entire reference gene list or are found primarily at the top or bottom of gene list. We performed GSEA using the Java GSEA implementation (supported by Java 8) to validate the enrichment analysis. Annotated gene sets c2.cp.kegg.v6.1.symbols.gmt, c5.bp.v6.1.symbols.gmt, c5.cc.v6.1.symbols.gmt, c5.mf.v6.1.0:0symbols.gmt (Version 6.1 of the Molecular Signatures Database) are chosen as the reference gene sets. FDR $<0.05$ is set as the cut-off criteria.

\section{Results}

\section{(1) Identification of DEGs in thyroid cancer}

As shown in Table 2, there are 340 DEGs in DTC-NTH; 162 DEGs in PDTC-NTH; 279 DEGs in PDTC-DTC; 484 DEGs in ATC-NTH; 5 DEGs in ATC-DTC; 276 DEGs in ATC-PDTC, respectively. The DEGs list of DTC-ALL is equal to that of DTCNTH. The DEGs list of PDTC-ALL is merged by that of PDTC-NTH and PDTC-DTC. And the DEGs list of ATC-All is merged by ATC-NTH, ATC-DTC, and ATC-PDTC. There are 340, 427, and 595 DEGs in DTC-ALL, PDTC-ALL, and ATC-ALL, respectively. Furthermore, in Fig. 1, it is found that 17 cross-expressed genes are identified in the occurrence of thyroid tumors (including DTC, PDTC, ATC) : ADH1B AGR3, C7, FAM3B, GPM6A, IPCEF, KLHDC8A, LONRF2, LRP1B, OGDHL, PLA2R1, PTX3, SMOC2, TENM1, TFF3, THRSP, TMEM139. Moreover, as shown in Figure S1 of the overlap gene lists circle figure, Blue represents the DEGs of DTC, and Green represents the DEGs of PDTC; Red represents the DEGs of ATC. The greater the number of purple links and the longer the dark orange arcs implies greater overlap among the input gene lists. It can be seen that PDTC owns longer dark orange color arc and more purple links, which means that there are more identical genes in the DEGs in development PDTC. We will further study the functional enrichment of DEGs of DTC, PDTC and ATC. 
Table 2

The DEGs information of DTC-ALL, PDTC-ALL, and ATC-ALL

\begin{tabular}{|lllllll|}
\hline \multirow{2}{*}{ Tumor type } & DTC-ALL & PDTC-ALL & \multicolumn{3}{c|}{ ATC-ALL } \\
\cline { 2 - 7 } & DTC-NTH & PDTC-NTH & PDTC-DTC & ATC-NTH & ATC-DTC & ATC-PDTC \\
\hline \multirow{2}{*}{ Numbers of DEGs } & 340 & 162 & 279 & 484 & 5 & 276 \\
\cline { 2 - 7 } & 340 & 427 & & 595 & & \\
\hline
\end{tabular}

\section{(2)Functional enrichment analysis of DEGs in DTC, PDTC, and ATC}

As shown in Fig. 2, it could be seen that the molecular function of enriched DEGs of DTC, PDTC, and ATC have a significant difference. Extracellular Matrix Organization is significantly enriched in DTC. While the following items in the PDTC are significantly enriched: Supramolecular Organization Fiber, Regulation of Cell adhesion, Positive Regulation of Cell Death, Regulation of Cascade of MAPK, negative Organization Regulation of Cellular Component. The enriched items in the ATC are: NABA MATRISOME ASSOCIATED, the PID INTEGRIN1 PATHEWAY, Extracellular Matrix Organization, Cell Regulation of adhesion, Blood Vessel Development, Regulation of positive Locomotion, Junction Cell Organization, Response to wounding, ossification, negative Regulation of Cell adhesion. While the following items are significantly enriched both in the PDTC and ATC: Supramolecular Fiber Organization, Regulation of Cell adhesion, Blood Vessel Development, Regulation of positive Locomotion, Signaling by Receptor Tyrosine Kinases. Moreover, functional enriched gene biological processes of DTC, PDTC, and ATC have obvious differences. Cellular component organization or biogenesis is obviously enriched in DTC. The biological adhesion, cellular component organization or biogenesis, biological regulation, signaling, negative regulation of biological process, cellular process, positive regulation of biological process, metabolic process, localization, regulation of biological process has obvious enrichment in PDTC. While the following items are enriched in ATC: developmental process, response to stimulus, biological adhesion, locomotion, cellular component organization or biogenesis, positive regulation of biological process, metabolic process, immune system process, multicellular organismal process. And biological adhesion, cellular component organization or biogenesis, positive regulation of biological process, the metabolic process are enriched in both PDTC and ATC.

Gene list enrichments are identified in the following ontology categories: TRRUST, DisGeNET, PaGenBase. The top few enriched clusters (one term per cluster) are shown in the Figure S2-4. As can be seen in Figure S2, the transcription factor (TF) enriched in ATC is significantly more than that in PDCT and DTC. And the transcription factor (TF) enriched in PDCT is more than that in DTC. The transcription factor (TF) of DTC is obviously enriched in VHL. The enriched transcription factor (TF) of PDTC: NFKB1, 53, 2F3, NF24, RELB. The transcription factor of ATC is significantly enriched in: NFKB1, SP1, RELA, TP53, JUN, ETS1; The common transcription factors (TF) enriched in PDTC and ATCare: NFKB1, SP1, STAT3, RELA, TP53, HDAC1, TFAP2A, E2F1, JUN.

Figure S5 shows the PPI network of DTC, which is enriched in cell morphogenesis involved in differentiation, chemotaxis, and taxis, with a significant difference. And the PPI network of DTC has 4 MCODEs. And in Figure S6., it can be seen that the enrichment of PPI network in PDTC are Regulation of Cytoskeleton Organization, Cell Division, positive kinase Activity Regulation, with significant difference. And the PPI network of PDTC has 4 MCODEs. Also, the Figure S7. Shows that the enrichment of PPI network in the ATC are extracellular matrix organization, extracellular structure organization, Extracellular matrix organization. And the PPI network of ATC has 11 MCODEs. The Table 3 summarizes the enrichment items of MCODEs in DTC, PDTC, and ATC, which could be found that chemotaxis may function in both DTC and PDTC. Moreover, the MCODE_2 in PDTC reveal sthat epidermal growth factor receptor signaling pathway might play an important role in development of PDTC.

Page 6/25 
Table 3

Summary of the enrichment items of MCODEs in DTC, PDTC, and ATC

\begin{tabular}{|c|c|c|c|c|c|c|c|c|c|}
\hline & \multicolumn{3}{|c|}{ DTC } & \multicolumn{3}{|c|}{ PDTC } & \multicolumn{3}{|c|}{ ATC } \\
\hline \multirow{3}{*}{$\begin{array}{l}\text { Enrich- } \\
\text { ment } \\
\text { items of } \\
\text { PPI }\end{array}$} & \multicolumn{3}{|c|}{ differentiation } & \multicolumn{3}{|c|}{$\begin{array}{l}\text { Regulation of Cytoskeleton } \\
\text { Organization }\end{array}$} & \multicolumn{3}{|c|}{ extracellular matrix organization } \\
\hline & \multicolumn{3}{|c|}{ chemotaxis } & \multicolumn{3}{|c|}{ Cell Division } & \multicolumn{3}{|c|}{ extracellular structure organization } \\
\hline & \multicolumn{3}{|c|}{ taxis } & \multicolumn{3}{|c|}{$\begin{array}{l}\text { positive kinase Activity } \\
\text { Regulation }\end{array}$} & \multicolumn{3}{|c|}{ Extracellular matrix organization } \\
\hline \multirow{9}{*}{$\begin{array}{l}\text { Numbers } \\
\text { of } \\
\text { MCODE } \\
\text { and } \\
\text { Enrich- } \\
\text { ment } \\
\text { items }\end{array}$} & \multirow{9}{*}{\multicolumn{2}{|c|}{$4 \quad$ MCODE_1 }} & \multirow{9}{*}{$\begin{array}{l}\text { G alpha (i) } \\
\text { signalling } \\
\text { events, } \\
\text { GPCR } \\
\text { ligand } \\
\text { binding, } \\
\text { positive } \\
\text { regulation } \\
\text { of } \\
\text { chemotaxis }\end{array}$} & \multirow[t]{9}{*}{4} & \multirow[t]{5}{*}{ MCODE_1 } & $\begin{array}{l}\text { negative } \\
\text { regulation of } \\
\text { dendritic cell }\end{array}$ & \multirow[t]{5}{*}{11} & \multirow[t]{2}{*}{ MCODE_1 } & $\begin{array}{l}\text { Chemokine } \\
\text { receptors bind } \\
\text { chemokines, }\end{array}$ \\
\hline & & & & & & $\begin{array}{l}\text { positive } \\
\text { regulation of } \\
\text { chemotaxis, } \\
\text { regulation of } \\
\text { dendritic cell }\end{array}$ & & & $\begin{array}{l}\text { chemokine- } \\
\text { mediated } \\
\text { signaling } \\
\text { pathway, } \\
\text { Chemokine } \\
\text { signaling } \\
\text { pathway }\end{array}$ \\
\hline & & & & & & process & & \multirow[t]{3}{*}{ MCODE_2 } & $\begin{array}{l}\text { GO in } \\
\text { Resolution of } \\
\text { Sister } \\
\text { Chromatid } \\
\text { Cohesion, }\end{array}$ \\
\hline & & & & & & & & & $\begin{array}{l}\text { Amplification } \\
\text { of signal from } \\
\text { the } \\
\text { kinetochores, }\end{array}$ \\
\hline & & & & & & & & & $\begin{array}{l}\text { Amplification } \\
\text { of signal from } \\
\text { unattached } \\
\text { kinetochores } \\
\text { via a MAD2 } \\
\text { inhibitory } \\
\text { signal }\end{array}$ \\
\hline & & & & & \multirow[t]{4}{*}{ MCODE_2 } & $\begin{array}{l}\text { epidermal } \\
\text { growth factor } \\
\text { receptor } \\
\text { signaling } \\
\text { pathway, }\end{array}$ & & \multirow[t]{4}{*}{ MCODE_3 } & $\begin{array}{l}\text { ADORA2B } \\
\text { mediated } \\
\text { anti- } \\
\text { inflammatory } \\
\text { cytokines } \\
\text { production, }\end{array}$ \\
\hline & & & & & & $\begin{array}{l}\text { ERBB } \\
\text { signaling } \\
\text { pathway, }\end{array}$ & & & \multirow{3}{*}{$\begin{array}{l}\text { Anti- } \\
\text { inflammatory } \\
\text { response } \\
\text { favouring } \\
\text { Leishmania } \\
\text { parasite } \\
\text { infection, } \\
\text { Leishmania } \\
\text { parasite } \\
\text { growth and } \\
\text { survival }\end{array}$} \\
\hline & & & & & & $\begin{array}{l}\text { Clathrin- } \\
\text { mediated } \\
\text { endocytosis }\end{array}$ & & & \\
\hline & & & & & & & & & \\
\hline
\end{tabular}




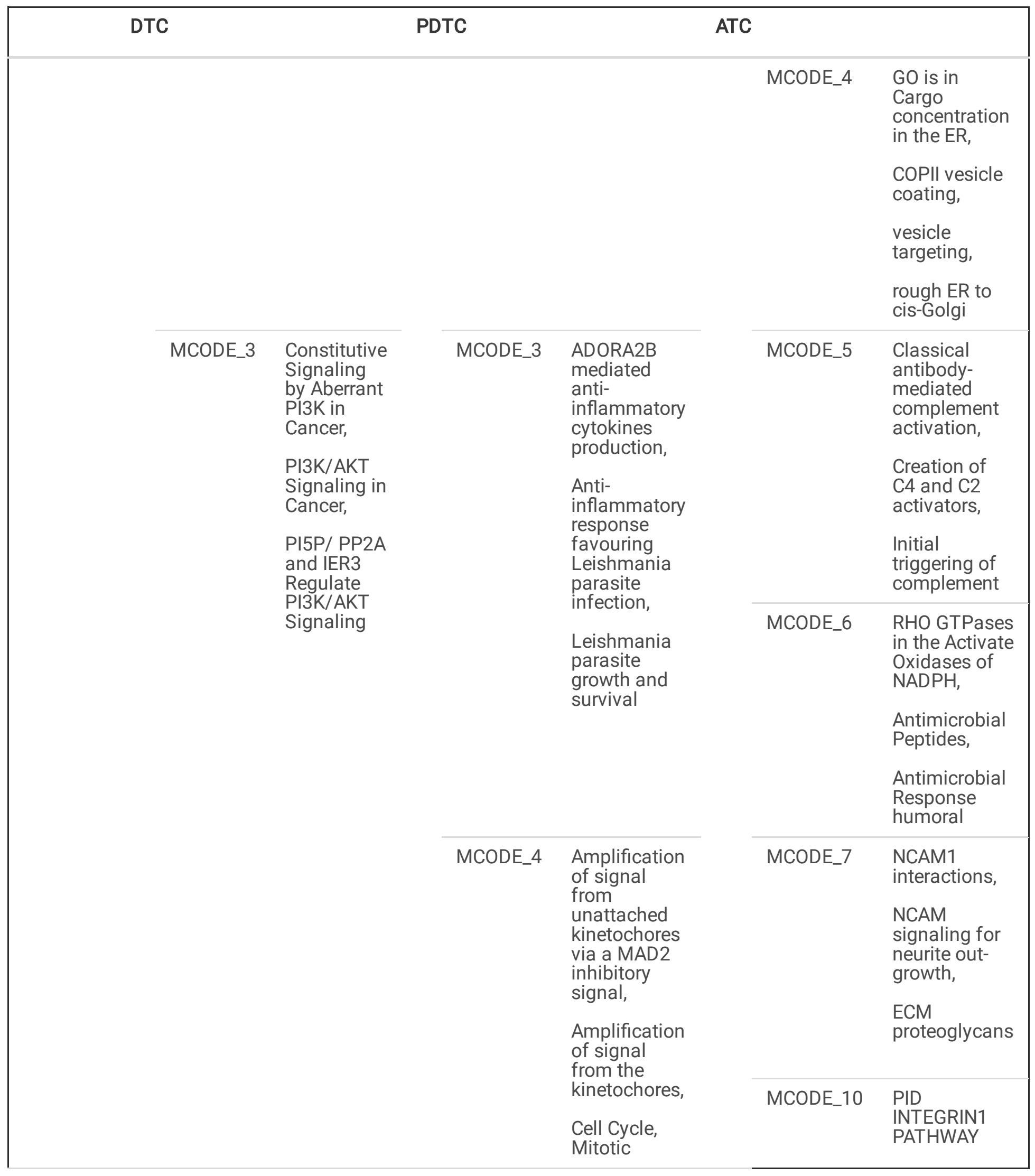

\section{『3षFunctional enrichment analysis of DEGs in PDTC}

As shown in Figure 3.A, it can be seen that PDTC-NTH and PDCT-DTC functional enrichment gene molecular functions are quite different. The enrichment of functional enrichment gene molecular functions in PDTC-NTH are: regulation of 
plasma membrane bounded cell projection organization, NABA CORE MATRISOME. While the following items are enriched PDTC-DTC: regulation of cytoskeleton organization, negative regulation of cellular component organization, regulation of mitotic cell cycle, regulated exocytosis, Hemostasis, positive regulation of cell death, positive regulation of protein kinase activity, nuclear division, cell division, Signaling by Receptor Tyrosine Kinases, negative regulation of cell cycle, neural precursor cell proliferation, response to oxidative stress.

The functional enriched gene biological processes of PDTC-NTH and PDCT-DTC have obvious differences and similarities, as shown in Figure 3.B. In PDTC-NTH, cellular process, cellular component organization or biogenesis, immune system process are significantly enriched. While the functional enriched gene biological processes of PDCTDTC is enriched in cellular process, cellular component organization or biogenesis, cell proliferation, regulation of biological process, signaling, negative regulation of biological process, cellular process, positive regulation of biological process, metabolic process, biological regulation, localization, the reproductive process.

When multiple gene lists are provided, all gene lists are merged into one list called "PDTC-ALL". PPI network and MCODE components are identified in the "PDTC-ALL"gene lists. All lists merged are colored by Counts (Figure S8.A) and Cluster (Figure S8.B), respectively. The PPI network of PDTC-ALL is enriched in Regulation of Cytoskeleton Organization, Cell Division, positive kinase Activity Regulation. And the Figure 4 show that PPI network of PDTC-ALL have 5 MCODEs. The enrichment of MCODE_1 in PDTC-ALL is in negative regulation of dendritic cell apoptotic process, positive regulation of chemotaxis, regulation of dendritic cell apoptotic process. While the following items are enriched in MCODE_2 in PDTCALL: epidermal growth factor receptor signaling pathway, ERBB signaling pathway, Clathrin-mediated endocytosis. And the enrichment of MCODE_3 in PDTC-ALL is in Post NMDA receptor activation events, Activation of NMDA receptors and postsynaptic events, glycolytic process. The MCODE_4 in PDTC-ALL is enriched in Amplification of signal from the kinetochores, Amplification of signal from unattached kinetochores via a MAD2 inhibitory signal, Cell Cycle, Mitotic.

Table 4 shows the core genes of PDTC-ALL in different MCODEs. The core genes of MCODE_1 in PDTC-ALL are: CXCL12, CCL21, CCL19, LPAR1, GABBR2, APP, MAT2A, MYH10, RAD2, ACTB, TUBB2B, KPNA5, EEF1A1, YWHAE, PAK3. The core genes of MCODE_2 in PDTC-ALL are: TGFB1, CTNNB1, GSK3B, MAP, GAP1, TWF1, EGF, HIP1, VAMP3, CD3D, SH3GL2. The core genes of MCODE_3 in PDTC-ALL are: TPR, PRKAA2, STIP1, PGM5, OGDHL, CFL1, CAMK1, UBA1, RAB11B, RAB5C, PRKAR1A. The core genes of MCODE_4 in PDTC-ALL are: AURKA, CENPA, NUF CLP1. The core genes of MCODE_5 in PDTC-ALL are: MEOX2, STX11, RUNX1T1. 
Summary of the core genes of PDTC-ALL in different MCODEs

\begin{tabular}{|c|c|c|}
\hline $\begin{array}{l}\text { Number of } \\
\text { MCODES in } \\
\text { PDTC-ALL }\end{array}$ & $\begin{array}{l}\text { Enrichment items of MCODEs in } \\
\text { PDTC-ALL }\end{array}$ & Core genes of MCODEs in PDTC-ALL \\
\hline MCODE_1 & $\begin{array}{l}\text { negative regulation of dendritic cell } \\
\text { apoptotic process, } \\
\text { positive regulation of chemotaxis, } \\
\text { regulation of dendritic cell apoptotic } \\
\text { process }\end{array}$ & $\begin{array}{l}\text { CXCL12, CCL21, CCL19, LPAR1, GABBR2, APP, MAT2A, } \\
\text { MYH10, RAD2, ACTB, TUBB2B, KPNA5, EEF1A1, } \\
\text { YWHAE, PAK3 }\end{array}$ \\
\hline MCODE_2 & $\begin{array}{l}\text { epidermal growth factor receptor } \\
\text { signaling pathway, } \\
\text { ERBB signaling pathway, } \\
\text { Clathrin-mediated endocytosis }\end{array}$ & $\begin{array}{l}\text { TGFB1, CTNNB1, GSK3B, MAP, GAP1, TWF1, EGF, HIP1, } \\
\text { VAMP3, CD3D, SH3GL2 }\end{array}$ \\
\hline MCODE_3 & $\begin{array}{l}\text { Post NMDA receptor activation events, } \\
\text { Activation of NMDA receptors and } \\
\text { postsynaptic events, } \\
\text { glycolytic process }\end{array}$ & $\begin{array}{l}\text { TPR, PRKAA2, STIP1, PGM5, OGDHL, CFL1, CAMK1, } \\
\text { UBA1, RAB11B, RAB5C, PRKAR1A }\end{array}$ \\
\hline MCODE_4 & $\begin{array}{l}\text { Amplification of signal from the } \\
\text { kinetochores, } \\
\text { Amplification of signal from } \\
\text { unattached kinetochores via a MAD2 } \\
\text { inhibitory signal, } \\
\text { Cell Cycle, } \\
\text { Mitotic }\end{array}$ & AURKA, CENPA, NUF CLP1 \\
\hline MCODE_5 & & MEOX2, STX11, RUNX1T1 \\
\hline
\end{tabular}

\section{『4囚Comprehensive co-Expression Network Analysis and Candidate Biomarker in PDTC}

In order to identify which gene may potentially play a biomarker in the development of PDTC, all significantly DEGs are used to construct gene co-expression network, as shown in Figure 5. The degree of a node describes the number of links one gene with others in the gene network. In the PDTC co-expression network, we founded the gene-expressions in PDTC are largely consistent with the previous study, such as the expression of CCND1 and KRAS. Some novel observations are also made in our study. Interestingly, the central of this network are CCNA2, EGF, which directly interacted with more than 20 neighboring genes. Furthermore, as shown in Figure 18, the top40 Hub genes are confirmed with MNC algorithm: CCNA2, EGF, AURKA, CCND1, CDC20, CD44, CTNNB1, ASPM, KIF4A, KIF20A, RACGAP1, KRAS, CKS2, DLGAP5, ACTB, PBK, NCAPG, RAD51AP1, NUSAP1, KIF15, NUF2, APP, KIAA0101, DEPDC1, MELK, ANLN, CDCA2, KIF14, SHCBP1, FANCI, OIP5, HGF, CXCL12, FAM83D, CDCA3, E2F8, CCNE2, STAT1, CD40, BCL2L1.

\section{Q5囚Relationship between the Expression of Hub genes and Clinical Features of thyroid cancer}


Further the top 40 Hub gene expression and survival analysis in thyroid cancer are checked in GEPIA. As shown in Figure 6, the expression of EGF, CCND1, DEPDC1, ANLN, HGF, and BCL2L1 in thyroid tissue and thyroid cancer tissue are significantly different, with a certain impact on the survival of thyroid tumors. The Figure shows the survival analysis curve of EGF, CCND1, DEPDC1, ANLN, HGF, and BCL2L1 in patients with thyroid cancer. Among them, the HR of BCL2L1 and CCND1 are less than 1.0, which are 0.32 and 0.26 respectively. The HR of EGF, DEPDC1, ANLN and HGF are greater than 1.0 , which are $5,2.69,2.99,3.19$ respectively.

By searching the human protein atlas database for evaluation of Hub gene protein expression, it was found that the protein expression of CCND1, DEPDC, ANLN, HGF, BCL2L1 in thyroid cancer and thyroid tissue had a certain difference, as shown in the Figure 8. The data of EGF was not retrieved, and EGFR was further searched and shown.

\section{(6) GSEA reveal a close relationship between hub genes and tumor proliferation}

Further the associated regulatory pathway analysis of DEGs of PDTC-NTH and PDTC-DTC are conducted in GSEA JAVA. There are 89/174 gene sets upregulated in phenotype PDTC-NTH. Two gene sets are enriched significantly at p-value < 1\%, including KEGG_THYROID_CANCER and KEGG_GLIOMA. And 11 gene sets are significantly enriched at $\mathrm{p}$-value $<5 \%$. The p-value of KEGG_P53_SIGNALING_PATHWAY is equal to 0.51. The Figure 9 shows the enrichment plot of these 12 KEGG pathway in PDTC_NTH.

There are 58/174 gene sets upregulated in phenotype PDTC_DTC. And 2 gene sets are significantly enriched at p-value $<5 \%$, including KEGG_TRYPTOPHAN_METABOLISM and KEGG_STARCH_AND_SUCROSE_METABOLISM. The Figure 10 shows the enrichment plot of these 2 KEGG pathway in PDTC_DTC.

\section{Discussion}

In recent years, studies have shown that thyroid cancer is a continuous process. With the accumulation of mutant genes, DTC can continue to progress into PDTC and ATC. In this study, it could be noted that Extracellular Matrix Organization is significantly enriched in DTC. While the following items are significantly enriched in both PDTC and ATC: Supramolecular Fiber Organization, Regulation of Cell adhesion, Blood Vessel Development, Regulation of positive Locomotion, Signaling by Receptor Tyrosine Kinases. From our research, it could be speculated that Regulation of Cell adhesion play an important role in the development of PDTC and ATC. The molecular functions of regulation of cell adhesion are enhanced in PDTC and ATC. In addition, the molecular functions of regulation of cell adhesion is obviously enhanced in ATC. Tumor cells shed from the original site and invade nearby normal tissues are the initial process of tumor metastasis. In this process, it is necessary to reduce the adhesion between tumor cells and enhance the adhesion between tumor cells and ECM, and to enhance the mobility of tumor cells so that the cells can invade adjacent normal tissues[23]. By adjusting the calcium sticky protein, tumor cells could reduce the adhesion between the cells, and then break through the basement membrane. Tumor cell surface Integrin protein (Integrin) is involved in tumor cells and ECM[24]. Generation of new blood vessels and tumor cells to vascular microenvironment interaction could be to promote adhesion and metastasis of tumor cells in the vascular endothelium. Enrichment of biological processes in PDTC and ATC are significantly enriched in biological processes related to biological adhesion, cellular component organization or biogenesis, positive regulation of biological process, and metabolic process. It can be seen that cell adhesion is an important step for the further development of thyroid tumors to malignant tumors. 
The biological behavior of tumor formation, development, infiltration and metastasis is a very complicated process, which is related to a series of serious disorder in the regulation of cell growth and proliferation. Cell growth and proliferation are under control in control and regulated by a number of factors, which include the more important growth factors (GF), growth factor receptors (GFR), signaling proteins and transcription factors(TF). The results of PPI network MCODEs of PDTC reveal that epidermal growth factor receptor signaling pathway might play an important role in development of PDTC. The core genes of MCODE_2 in PDTC are: EGF, CD3D, HIP1, SH3GL2, VAMP3, CTNNB1, TGFB1, GSK3B, MAP2K2, IQGAP1, GSN, TWF1. And 6 hub-genes were obtained in the development of PDTC through the algorithm of MNC, including EGF, CCND1, DEPDC1, ANLN, HGF, BCL2L1. Furthermore, the survival analysis of top 40 Hub genes in thyroid cancer were analyzes in GEPIA. As shown in Figs. 5 and 6, the expression of EGF, CCND1, DEPDC1, ANLN, HGF, and BCL2L1 in thyroid tissue and thyroid cancer tissue are significantly different, with a certain impact on the survival of thyroid tumors. EGF is a strong cell division promoting factor, which is closely related to the occurrence and development of tumors. The activation of EGF-EGFR are accelerating cell proliferation, involved in the regulation of autophagy activity. In the meantime it will promote the malignant transformation of tumors by regulating the cell adhesion through epithelial-mesenchymal cell turnover (EMT) [25]. Ota et.al[26] have found that heparin-binding EGFlike growth factor activity enhanced invasion and metastasis of thyroid cancer; the study of Xue indicates MiR-200 mediated by EGF/EGFR signaling regulates epithelial-mesenchymal transition in anaplastic thyroid cancer[27]. EGFRmediated downstream signaling pathways mainly include PI3K/AKT/mTOR signaling pathway, JAK/STAT signaling pathway and MAPK/P38 signaling pathway. Hepatocyte growth factor (HGF) is ligand of tyrosine kinase receptor (epithelial mesenchymal conversion factor). And the combination of the two can induce the activation of carcinogenic pathways, promote angiogenesis and tumor metastasis[28]. Knauf et al.[29] found that HGF/Met activation mediates the resistance of mouse anaplastic thyroid cancer to BRAF inhibition, but its mechanism of action is not yet clear. In addition, both EGF and HGF can induce the activation of $\beta$-catenin and promote cell migration[30, 31], thereby activating the Wnt/ $\beta$-catenin signaling pathway.. The abnormal activation of Wnt / $\beta$-catenin signaling pathway is usually caused by somatic mutations, and its hot-spot mutation genes include: CTNNB1, KRAS, BRAF, NRAS, ERBB2, MET, PIK3CA, JAK1, etc[32]. CTNNB1 also plays an important role in epidermal growth factor receptor signaling pathway. However, the mechanism of EGF-EGFR and HGF in the malignant transformation of thyroid tumors needs further study.

Moreover, tumors are a type of diseases that cause uncontrolled cell growth and cell cycle disorders due to multi-gene mutation. Among them, a series of factors jointly regulate the cell cycle, and the role of cyclin is the most important, which is close related to regulate the growth of tumors. Cyclin 1 (CCND1) can combine with cyclin-dependent kinases (CDKs), such as CDK4 or CDK6, to form a complex and promote the cell cycle from G1 to S Phase transformation[33]. Sun et al. found that the silencing of E2F8 significantly inhibited the proliferation of PTC cells in vivo and in vitro by down-regulating CCND1, leading to G1 phase arrest[34]. In addition, CCND1 also plays a pivotal role in promoting tumor invasion and metastasis. Guo et al.[35] found that long-chain non-coding RNA NR2F1-AS1 promotes the proliferation and migration of thyroid cancer cells by regulating the miRNA-338-3p/CCND1 axis. Furthermore, anillin (ANLN) is a gene encoding actin binding protein, which is considered to be an important part of cell division. Recent studies have found that ANLN is involved in the occurrence and development of a variety of malignant tumors, and can promote tumor cell growth, migration and invasion. In bladder cancer, down-regulation of ANLN expression significantly reduces cell proliferation, migration and invasion capabilities[36]. Wang et al.[37] found that ANLN is involved in the progression of pancreatic cancer by regulating the EZH2/miR-218-5p/LASP1 signal axis. Although there have been findings pointing to a contribution of ANLN to the development of cancer, the association of ANLN and thyroid cancer remains not understood. Recently, the impact of DEPDC1 on the biology of various malignant tumors has attracted the attention of many scholars. Human-derived DEPDC1 is enhanced by mitotic cell cycle thereby resulting in cause -related genes tumorigenesis[38]. And BCL2L1 belongs to BCL2 Family. It can form heterodimers with Bax and Bak in cells, thereby inhibiting the opening of VDAC channels and the occurrence of apoptotic reactions[39]. Therefore, BCL2L1 is an

Page $12 / 25$ 
important anti-apoptotic factor. However, the role and mechanism of BCL2L1 in the process of malignant transformation of thyroid tumors is remains to be further studied.

\section{Conclusions}

This study analyzed multiple genomes of DTC, PDTC, ATC and found the gene expression characteristics of these three types of thyroid cancer. Results confirmed that in the process of malignant transformation of thyroid cancer, the MF, BO, and KEGG pathways involved has changed, especially the activation of cytoskeleton and extracellular matrix tissue, which promote the malignant transformation of thyroid tumors. Furthermore, six hub-genes, especially EGF and CCND1, can become potential biomarkers of PDTC. We suspect that these six hub-genes may be potential biomarkers of PDTC. Exploration of genomes of DTC, PDTC, ATC provides ideas for a comprehensive understanding of the development of PDTC. In addition, further researches on these hub genes are needed.

\section{Declarations}

\section{Acknowledgments}

The authors thank the teachers of Department of Thyroid Surgery, The First Affiliated Hospital of Zhengzhou University for suggestions.

\section{Funding}

This study was supported by College Scientific and Technological Innovation Team Project of Henan Province (No. 19IRTSTHN002), The Thousand Talents Science and Technology Innovation Leading Talents Subsidy Project of Central Plains (194200510011), Major Scientific Research Projects of Traditional Chinese Medicine in Henan Province (No.2021ZYZD14), Cultivation of Young and Middle-aged Health Science and Technology Innovation Leading Talents in Henan Province(YXKC2020015).

\section{Authors' contributions}

Study design: DetaoYin. Data collection: XinChen and RunshengMa. Analysis and interpretation of data: XinChen, HongqiangLi1, and YifengTang. Literature search and organization of figure and table: XinChen, RunshengMa, HongqiangLi, YifengTang. Study supervision: DetaoYin. All authors read and approved the final manuscript.

\section{Availability of data and materials}

All data generated or analyzed during this study are included in this published article.

\section{Ethics approval and consent to participate}

Not applicable

\section{Consent for publication}


All of the authors of this article have read and approved the final version including details and images. Written informed consent for the publication has been obtained from all of the authors.

\section{Competing interests}

All of the authors of this article declare that they have no competing interests.

\section{References}

1. La Vecchia C, Malvezzi M Fau - Bosetti C, Bosetti C Fau - Garavello W, Garavello W Fau - Bertuccio P, Bertuccio P Fau - Levi F, Levi F Fau - Negri E, et al. Thyroid cancer mortality and incidence: a global overview.

2. Park S, Oh CM, Cho H, Lee JY, Jung KW, Jun JK, et al. Association between screening and the thyroid cancer "epidemic" in South Korea: evidence from a nationwide study.

3. Roman BR, Morris Lg Fau - Davies L, Davies L. The thyroid cancer epidemic, 2017 perspective.

4. Siegel RL, Miller KD, Jemal AA-O. Cancer statistics, 2018.

5. Liu Z, Hou P, Ji M, Guan H, Studeman K, Jensen K, et al. Highly prevalent genetic alterations in receptor tyrosine kinases and phosphatidylinositol 3-kinase/akt and mitogen-activated protein kinase pathways in anaplastic and follicular thyroid cancers. J Clin Endocrinol Metab. 2008; 93: 3106-16.

6. Wang Y, Hou P, Yu H, Wang W, Ji M, Zhao S, et al. High prevalence and mutual exclusivity of genetic alterations in the phosphatidylinositol-3-kinase/akt pathway in thyroid tumors. J Clin Endocrinol Metab. 2007; 92: 2387-90.

7. Ricarte-Filho JC, Ryder M, Chitale DA, Rivera M, Heguy A, Ladanyi M, et al. Mutational profile of advanced primary and metastatic radioactive iodine-refractory thyroid cancers reveals distinct pathogenetic roles for BRAF, PIK3CA, and AKT1. Cancer Res. 2009; 69: 4885-93.

8. Hou P, Liu D, Xing M. Functional characterization of the T1799-1801del and A1799-1816ins BRAF mutations in papillary thyroid cancer. Cell Cycle. 2007; 6: 377-9.

9. Trovisco V, Soares P, Preto A, de Castro IV, Lima J, Castro P, et al. Type and prevalence of BRAF mutations are closely associated with papillary thyroid carcinoma histotype and patients' age but not with tumour aggressiveness. Virchows Arch. 2005; 446: 589-95.

10. Xing M, Westra WH, Tufano RP, Cohen Y, Rosenbaum E, Rhoden KJ, et al. BRAF mutation predicts a poorer clinical prognosis for papillary thyroid cancer. J Clin Endocrinol Metab. 2005; 90: 6373-9.

11. Knauf JA, Ma X, Smith EP, Zhang L, Mitsutake N, Liao XH, et al. Targeted expression of BRAFV600E in thyroid cells of transgenic mice results in papillary thyroid cancers that undergo dedifferentiation. Cancer Res. 2005; 65: 423845.

12. Abubaker J, Jehan Z, Bavi P, Sultana M, Al-Harbi S, Ibrahim M, et al. Clinicopathological analysis of papillary thyroid cancer with PIK3CA alterations in a Middle Eastern population. J Clin Endocrinol Metab. 2008; 93: 611-8.

13. Xing M. Molecular pathogenesis and mechanisms of thyroid cancer. Nat Rev Cancer. 2013; 13: 184-99.

14. Kennedy AL, Morton JP, Manoharan I, Nelson DM, Jamieson NB, Pawlikowski JS, et al. Activation of the PIK3CA/AKT pathway suppresses senescence induced by an activated RAS oncogene to promote tumorigenesis. Mol Cell. 2011; 42: 36-49.

15. Tirrò EA-O, Martorana F, Romano C, Vitale SR, Motta G, Di Gregorio S, et al. Molecular Alterations in Thyroid Cancer: From Bench to Clinical Practice. LID - 10.3390/genes10090709 [doi] LID - 709.

16. Xing M. Molecular pathogenesis and mechanisms of thyroid cancer. 
17. Chen N, Yin D, Lun B, Guo XJBr. LncRNA GAS8-AS1 suppresses papillary thyroid carcinoma cell growth through the miR-135b-5p/CCND2 axis. 2019; 39.

18. Zhang L, Wang L, Lei M, Ma R, Yu F, Liu C, et al. Generation and identification of a thyroid cancer cell line with stable expression of CCDC67 and luciferase reporter genes. 2019; 18: 4495-502.

19. Kazaure HS, Roman SA, Sosa JA. Insular thyroid cancer: a population-level analysis of patient characteristics and predictors of survival. Cancer. 2012; 118: 3260-7.

20. Patel KN, Shaha AR. Poorly differentiated thyroid cancer. Current opinion in otolaryngology \& head and neck surgery. 2011; 22: 121-6.

21. Slatko BE, Gardner AF, Ausubel FM. Overview of Next-Generation Sequencing Technologies.

22. Gauthier J, Vincent AT, Charette SJ, Derome N. A brief history of bioinformatics.

23. He X, Lee $B$, Jiang Y. Cell-ECM Interactions in Tumor Invasion.

24. Li ZH, Zhou Y, Ding YX, Guo QL, Zhao L. Roles of integrin in tumor development and the target inhibitors.

25. Sheng W, Chen C, Dong M, Wang G, Zhou J, Song H, et al. Calreticulin promotes EGF-induced EMT in pancreatic cancer cells via Integrin/EGFR-ERK/MAPK signaling pathway. Cell Death Dis. 2017; 8: e3147.

26. Ota I, Higashiyama S Fau - Masui T, Masui T Fau - Yane K, Yane K Fau - Hosoi H, Hosoi H Fau - Matsuura N, Matsuura N. Heparin-binding EGF-like growth factor enhances the activity of invasion and metastasis in thyroid cancer cells.

27. Xue L, Su D, Li D, Gao W, Yuan R, Pang W. Retraction Note to: miR-200 Regulates Epithelial-Mesenchymal Transition in Anaplastic Thyroid Cancer via EGF/EGFR Signaling.

28. Suárez-Causado A, Caballero-Díaz D, Bertrán E, Roncero C, Addante A, García-Álvaro M, et al. HGF/c-Met signaling promotes liver progenitor cell migration and invasion by an epithelial-mesenchymal transition-independent, phosphatidyl inositol-3 kinase-dependent pathway in an in vitro model.

29. Knauf JA, Luckett KA, Chen KY, Voza F, Socci ND, Ghossein R, et al. Hgf/Met activation mediates resistance to BRAF inhibition in murine anaplastic thyroid cancers.

30. Liu L, Yang Y, Liu S, Tao T, Cai J, Wu J, et al. EGF-induced nuclear localization of SHCBP1 activates $\beta$-catenin signaling and promotes cancer progression, Suppression of Wnt/ $\beta$-catenin signaling by EGF receptor is required for hair follicle development, The critical role of EGF- $\beta$-catenin signaling in the epithelial-mesenchymal transition in human glioblastoma , EGF Inhibits Wnt/ $\beta$-Catenin-Induced Osteoblast Differentiation by Promoting $\beta$-Catenin Degradation, FBXW2 suppresses migration and invasion of lung cancer cells via promoting $\beta$-catenin ubiquitylation and degradation, $\beta$-Catenin nuclear localization positively feeds back on EGF/EGFR-attenuated AJAP1 expression in breast cancer, TMEPAI/PMEPA1 inhibits Wnt signaling by regulating $\beta$-catenin stability and nuclear accumulation in triple negative breast cancer cells, Elevated $\beta$-catenin pathway as a novel target for patients with resistance to EGF receptor targeting drugs, Epidermal growth factor can signal via $\beta$-catenin to control proliferation of mesenchymal stem cells independently of canonical Wnt signalling, WNT7A Promotes EGF-Induced Migration of Oral Squamous Cell Carcinoma Cells by Activating $\beta$-Catenin/MMP9-Mediated Signaling.

31. Chaudhary P, Malhotra SS, Babu GS, Sobti RC, Gupta SA-O. HGF promotes HTR-8/SVneo cell migration through activation of MAPK/PKA signaling leading to up-regulation of WNT ligands and integrins that target $\beta$-catenin.

32. Taciak B, Pruszynska I, Kiraga L, Bialasek M, Krol M. Wnt signaling pathway in development and cancer. LID 10.26402/jpp.2018.2.07 [doi].

33. Ramos-García PA-O, González-Moles M, Ayén Á, González-Ruiz L, Gil-Montoya JA, Ruiz-Ávila I. Predictive value of CCND1/cyclin D1 alterations in the malignant transformation of potentially malignant head and neck disorders: Systematic review and meta-analysis. 
34. Sun J, Shi R, Zhao S, Li X, Lu S, Bu H, et al. E2F8, a direct target of miR-144, promotes papillary thyroid cancer progression via regulating cell cycle.

35. Guo F, Fu Q, Wang Y, Sui GA-O. Long non-coding RNA NR2F1-AS1 promoted proliferation and migration yet suppressed apoptosis of thyroid cancer cells through regulating miRNA-338-3p/CCND1 axis.

36. Sugawara S, Yamada Y, Arai T, Okato A, Idichi T, Kato M, et al. Dual strands of the miR-223 duplex (miR-223-5p and miR-223-3p) inhibit cancer cell aggressiveness: targeted genes are involved in bladder cancer pathogenesis.

37. Wang A, Dai H, Gong Y, Zhang C, Shu J, Luo Y, et al. ANLN-induced EZH2 upregulation promotes pancreatic cancer progression by mediating miR-218-5p/LASP1 signaling axis.

38. Mi Y, Zhang C, Bu Y, Zhang Y, He L, Li H, et al. DEPDC1 is a novel cell cycle related gene that regulates mitotic progression.

39. Huang H, Hu X Fau - Eno CO, Eno Co Fau - Zhao G, Zhao G Fau - Li C, Li C Fau - White C, White C. An interaction between $\mathrm{Bcl}-\mathrm{xL}$ and the voltage-dependent anion channel (VDAC) promotes mitochondrial Ca2+ uptake.

\section{Figures}




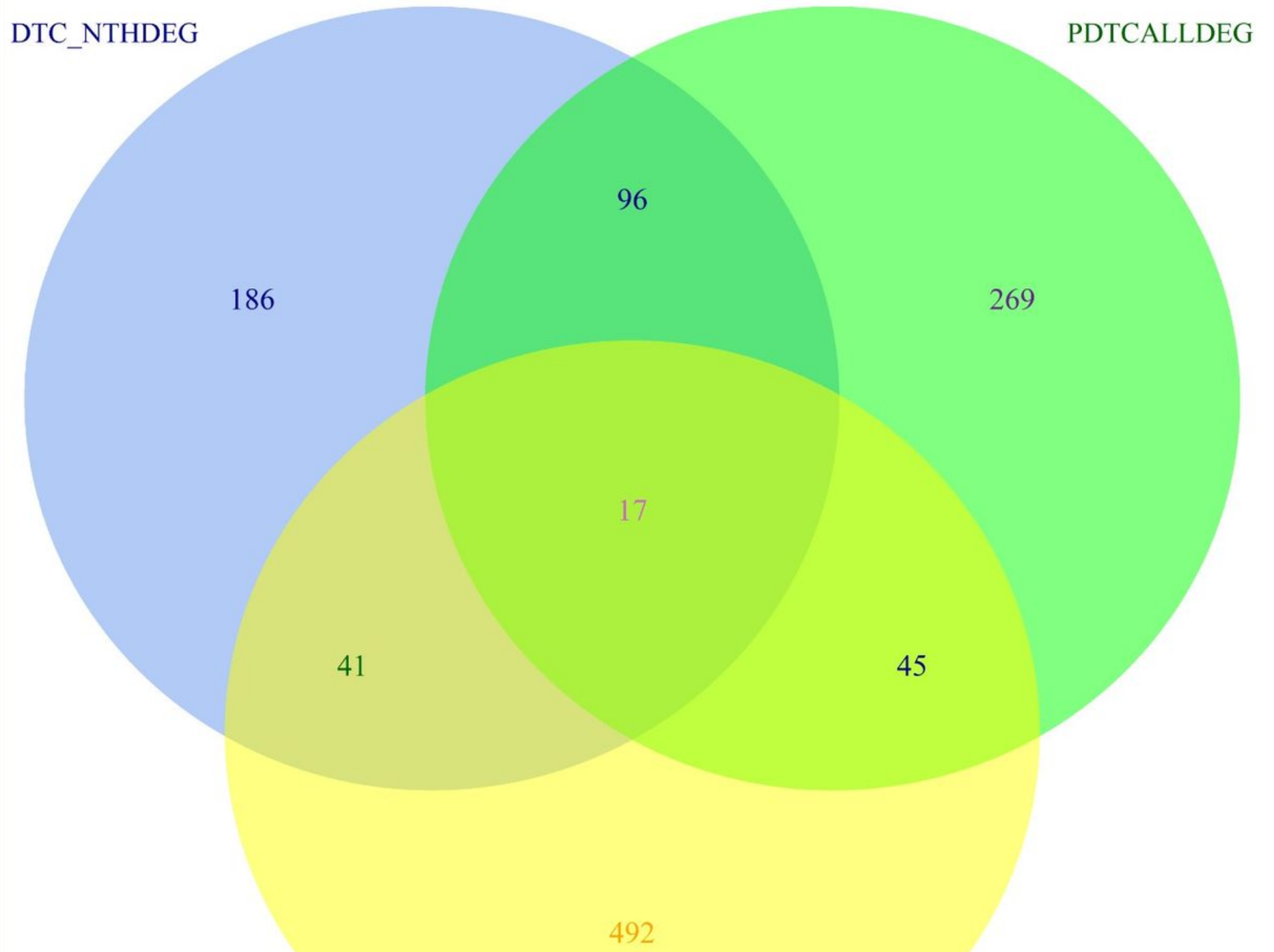

\section{ATCALLDEG}

Figure 1

Venn figures of DEG of DTC-ALL, PDTC-ALL, and ATC-ALL. 

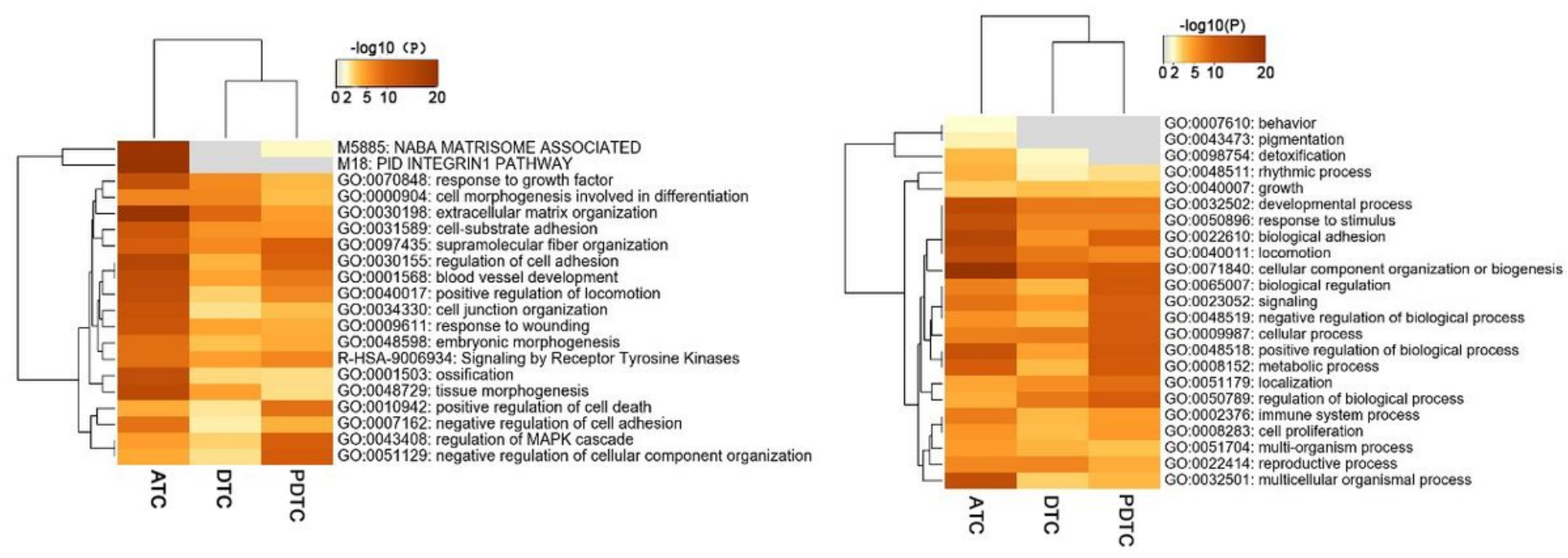

Figure 2

Heatmap of enriched terms across the top-level Gene Ontology molecular function(A) and biological processes(B) among DTC, PDTC, and ATC, colored by p-values.
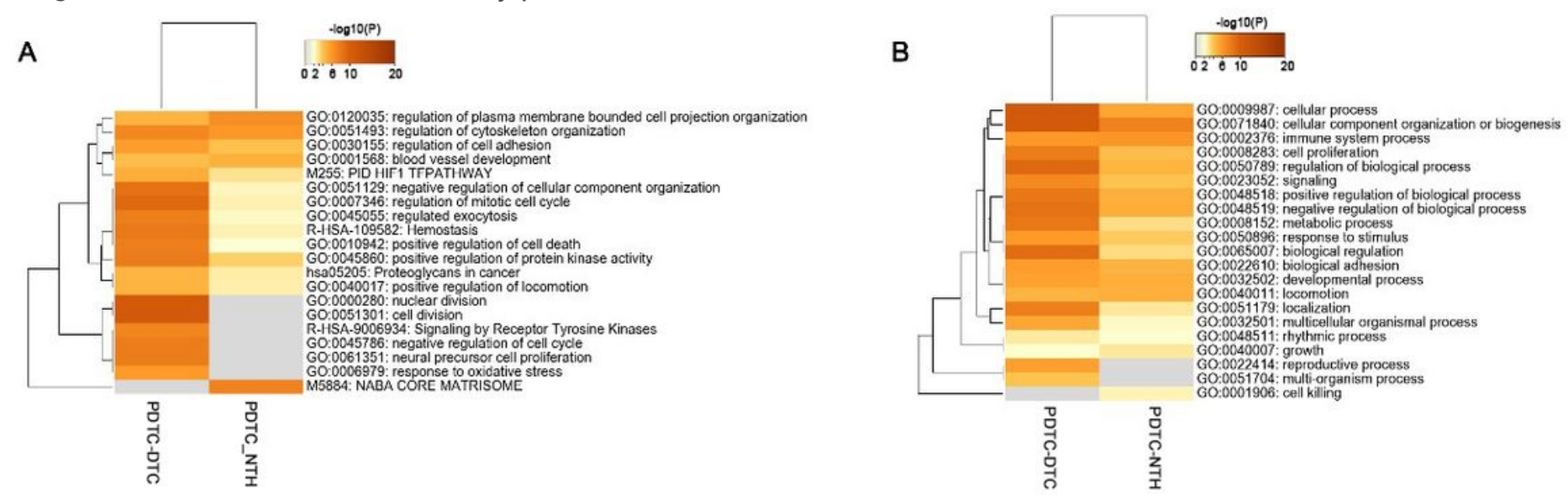

Figure 3

Heatmap of enriched terms across the top-level Gene Ontology molecular function(A) and biological processes(B) among PDTC-NTH and PDTC-DTC, colored by p-values. 

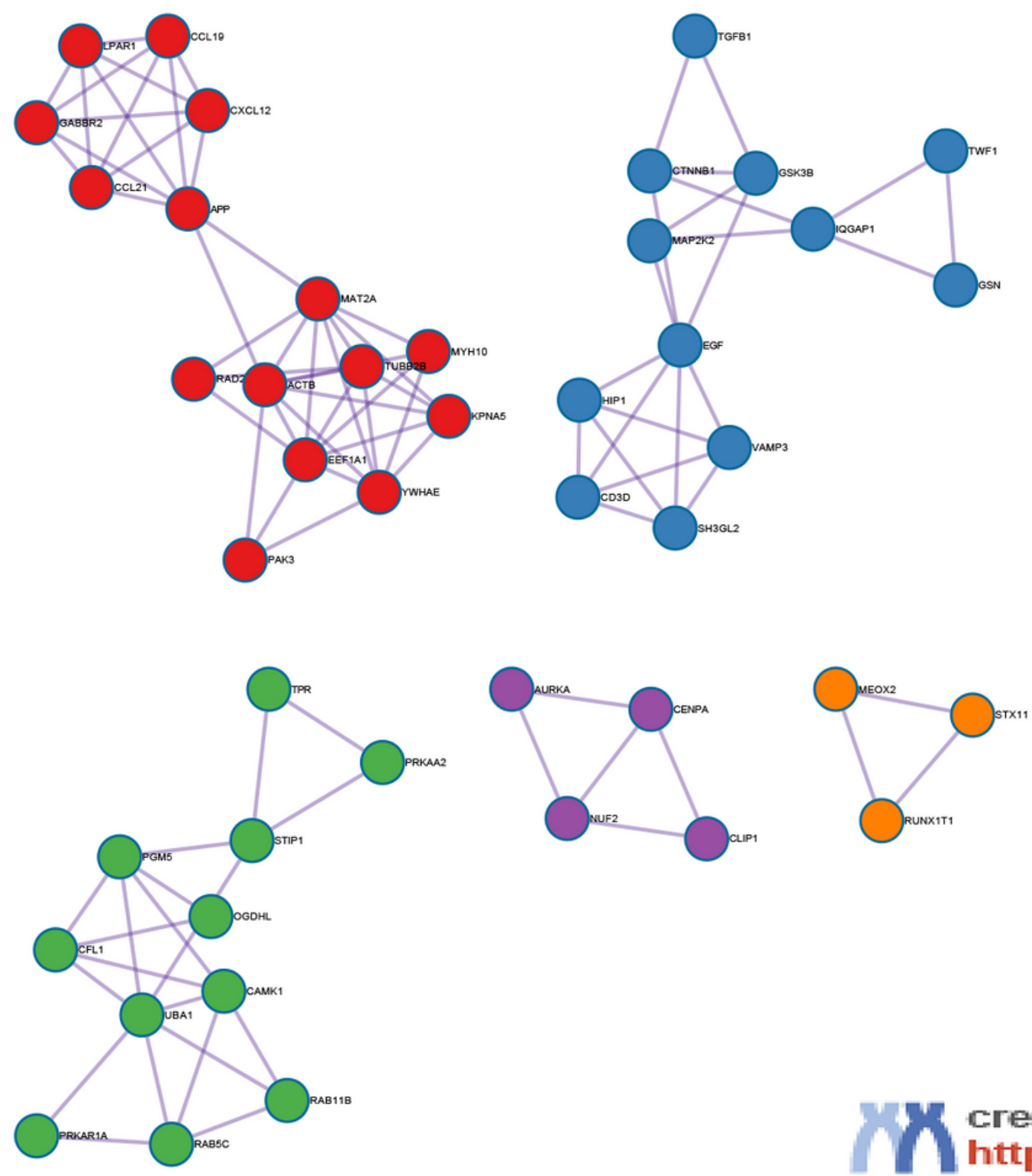

\section{Figure 4}

MCODE components identified in the gene lists of PDTC-ALL. All lists merged Colored by Counts. Network nodes are displayed as pies. Color code for pie sector represents a gene list and is consistent with the colors used for table rows in slide. Each MCODE network is assigned a unique color. 


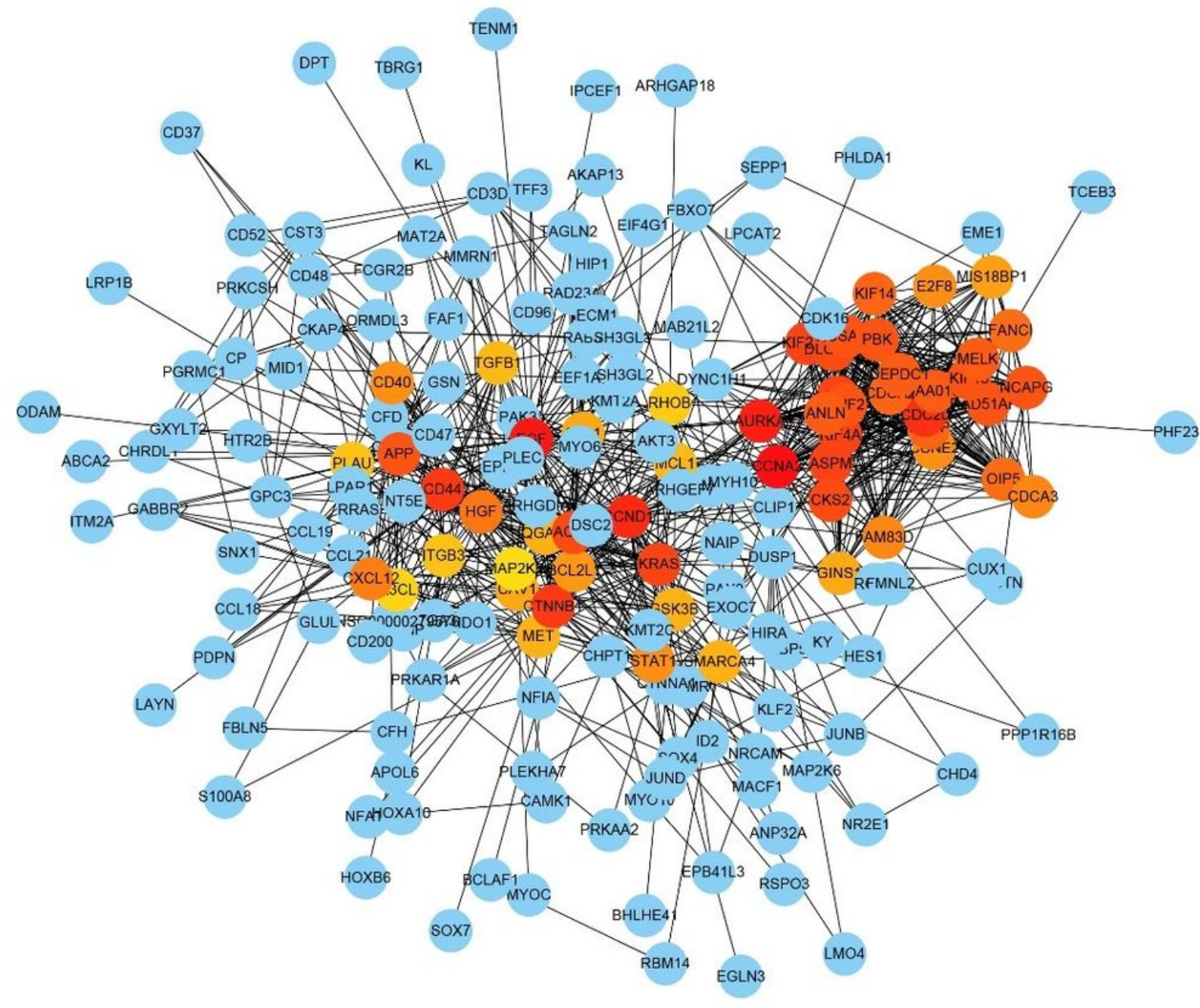

Figure 5

Protein-protein interaction network identified in the gene lists of PDTC-ALL. The Hub genes were calculated by method of MNC. And the Hub genes were labeled as warm colors, colored by p-value, where terms containing more genes tend to have a more significant $p$-value. 

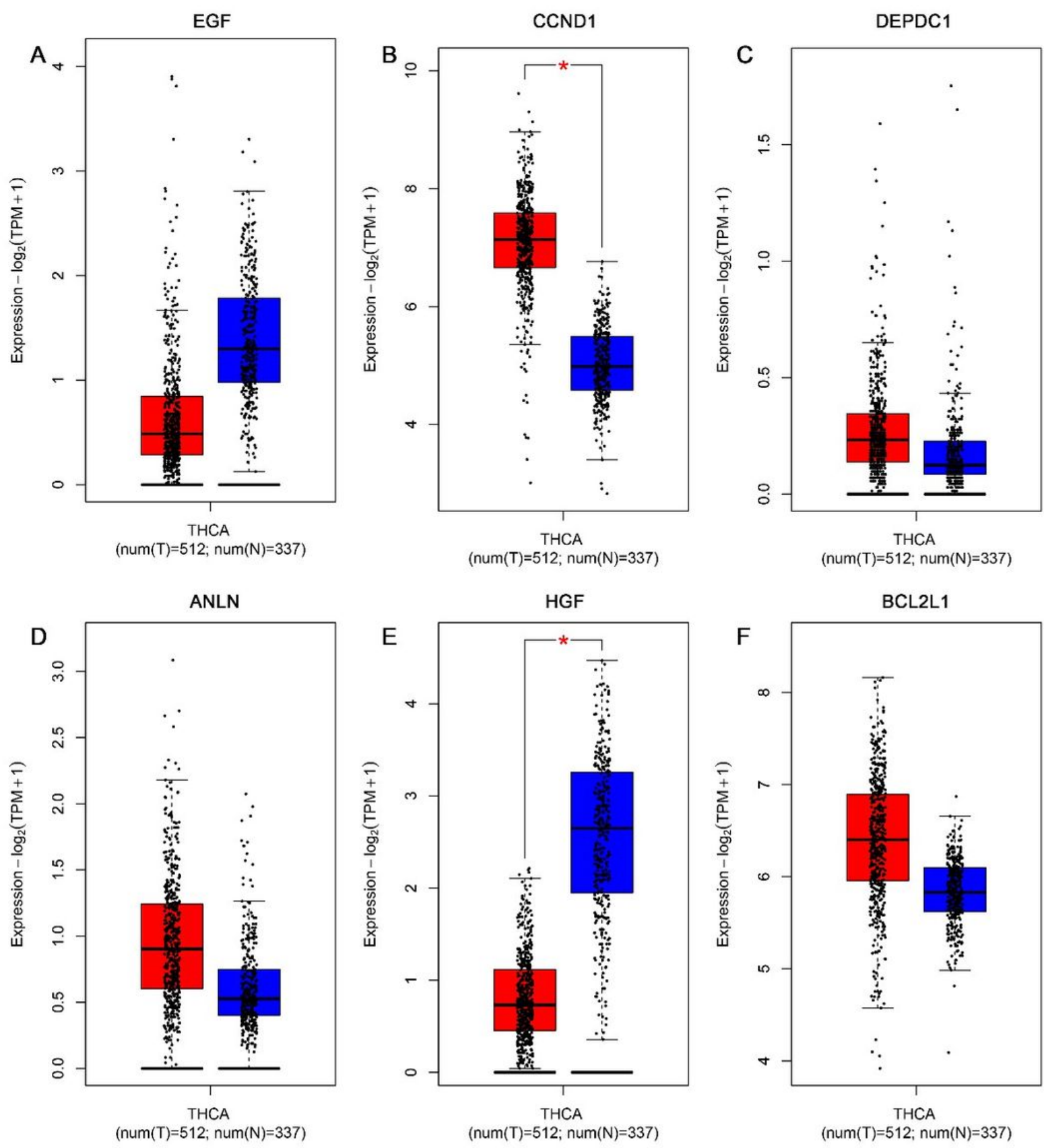

Figure 6

Gene expression level of EGF, CCND1, DEPDC1, ANLN, HGF, BCL2L1 in thyroid tumor and normal thyroid tissue. 

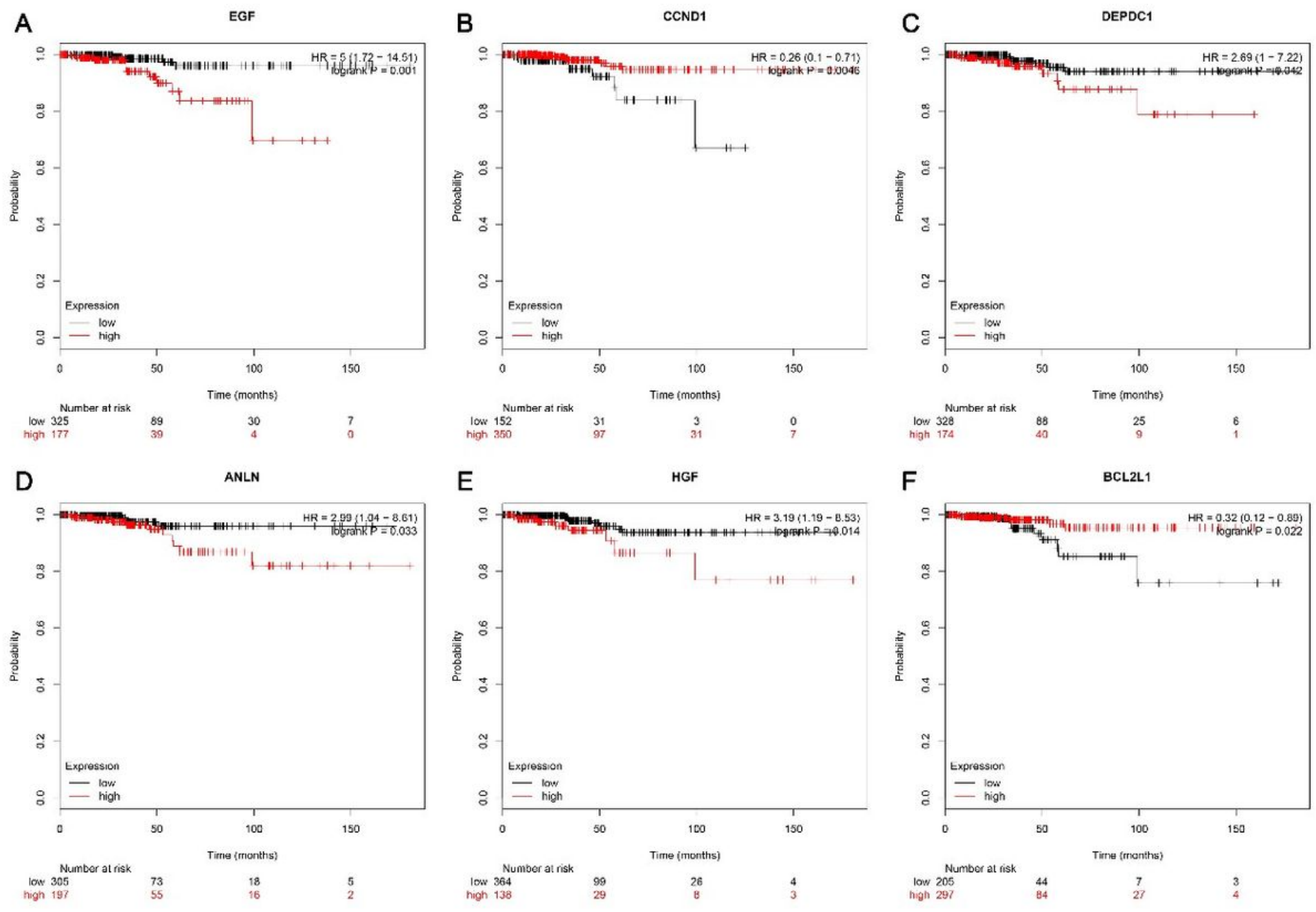

Figure 7

Survival analysis figure of EGF, CCND1, DEPDC1, ANLN, HGF, BCL2L1. 


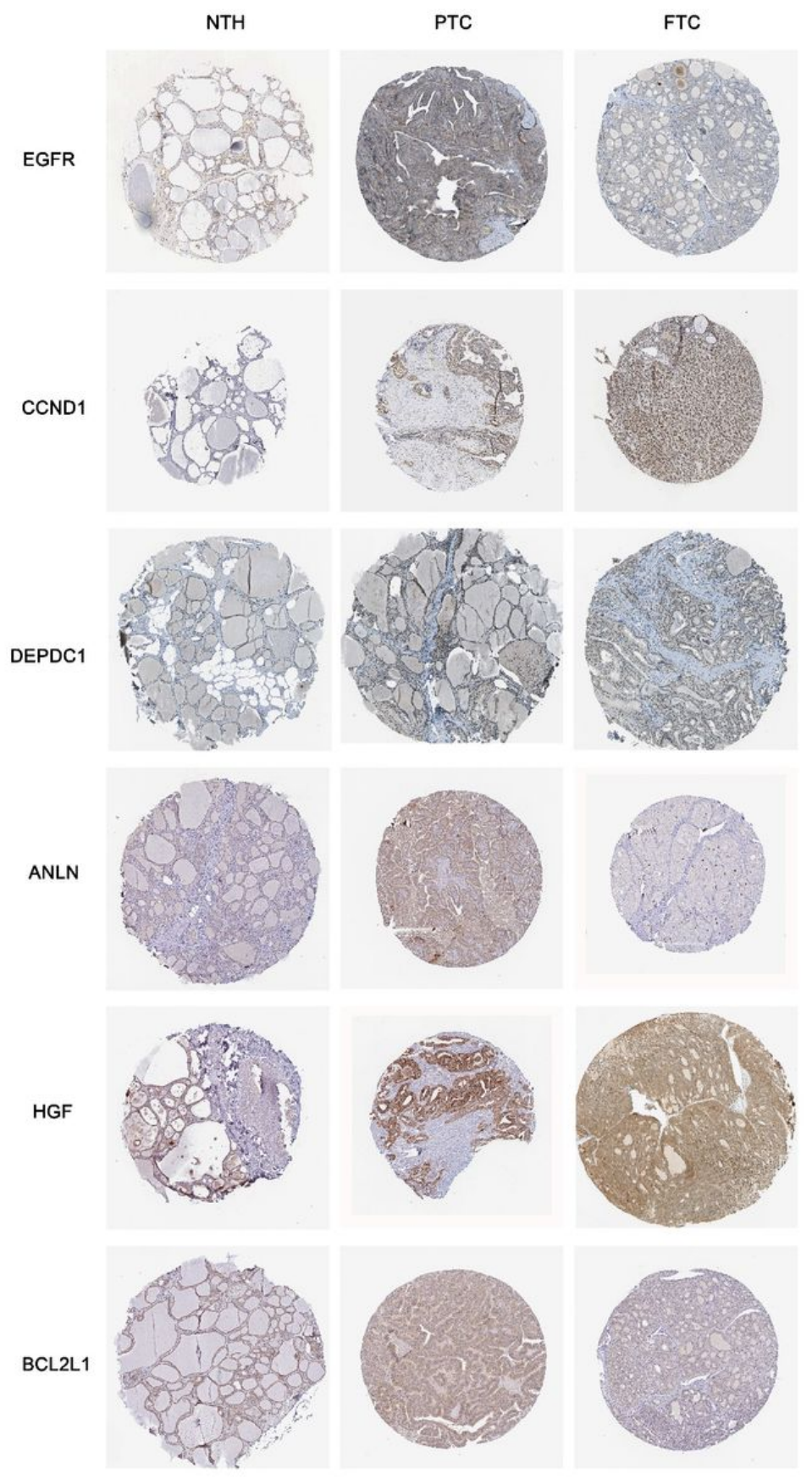

Figure 8

Immunohistochemical image of EGFR, CCND1, DEPDC1, ANLN, HGF, BCL2L1 among NTH, PTC, and FTC. 


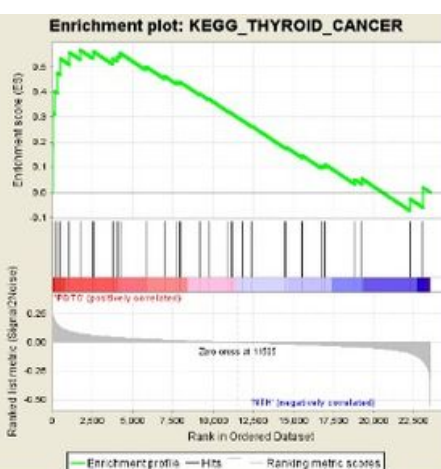

Enrichment plot: KEGG_O_GLYCAN_BIOSYNTHESIS
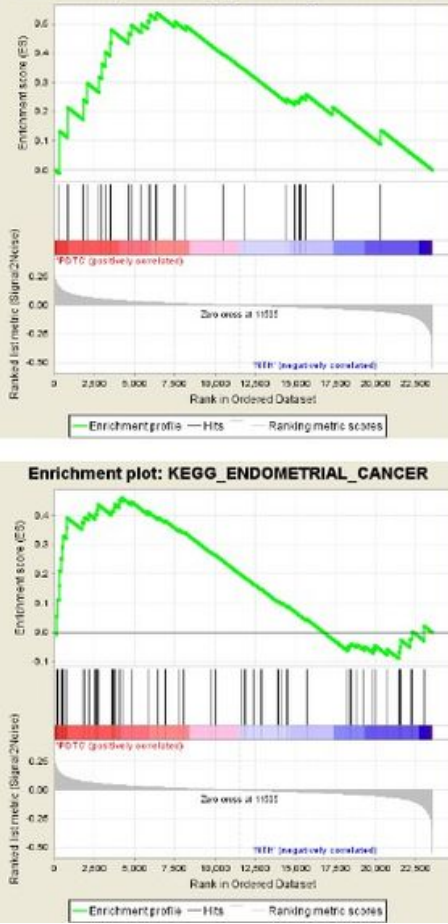

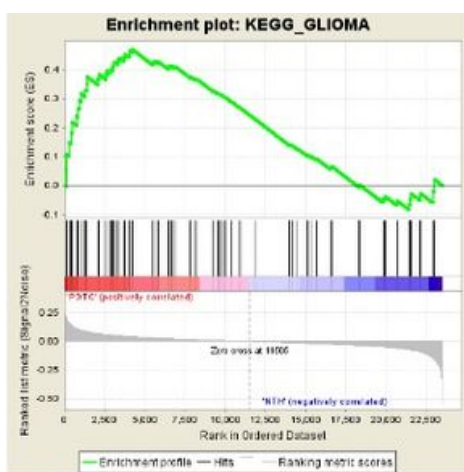

Enrichment plot: KEGG_ENDOMETRIAL_CANCER
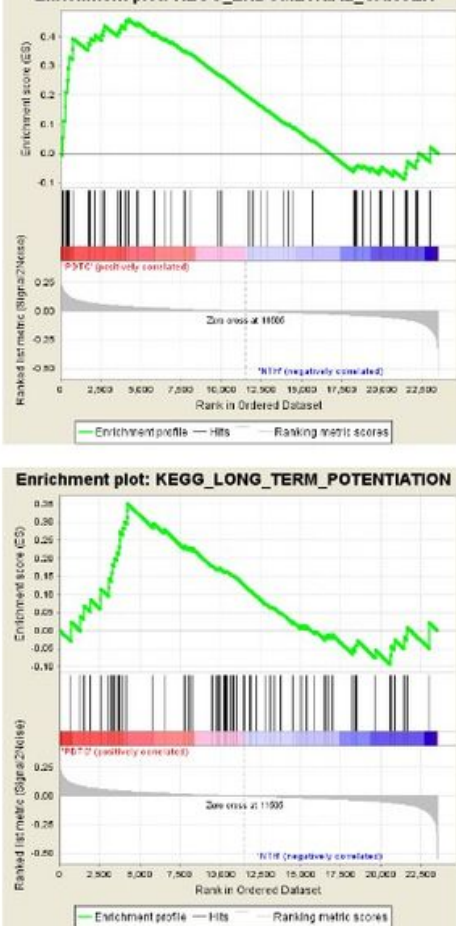

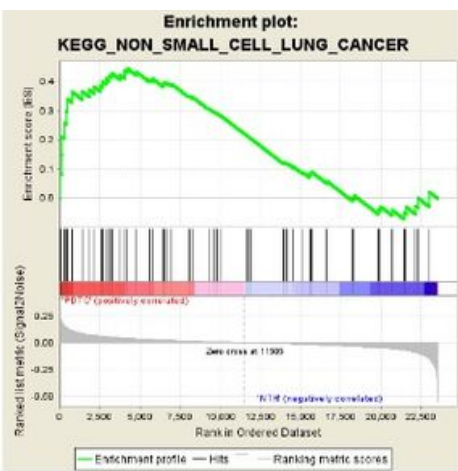

Enrichment plot:

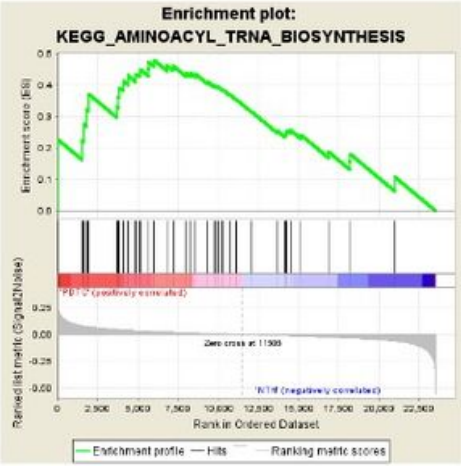

Enrichment plot: KEGG_GNRH_SIGNALING_PATHWAY
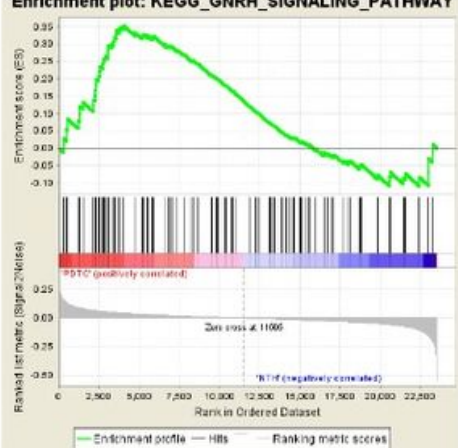

Enrichment plot: KEGG_OOCYTE_MEIOSIS

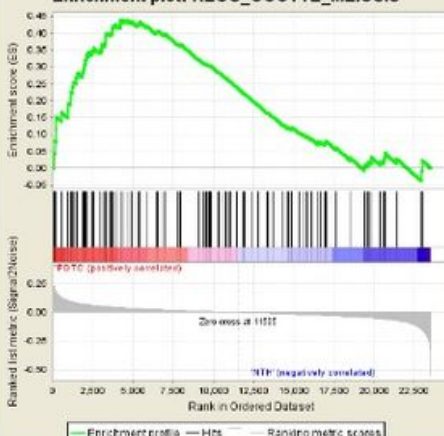

Enrichment plot: KEGG_P53_SIGNALING_PATHWAY
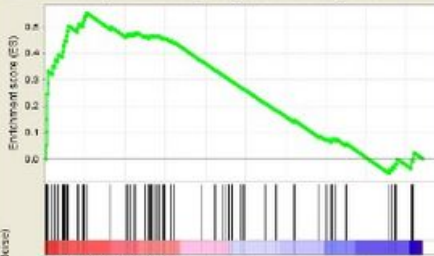

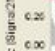

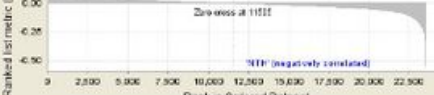

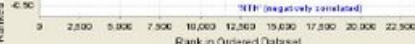

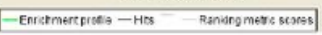

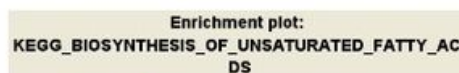
DS

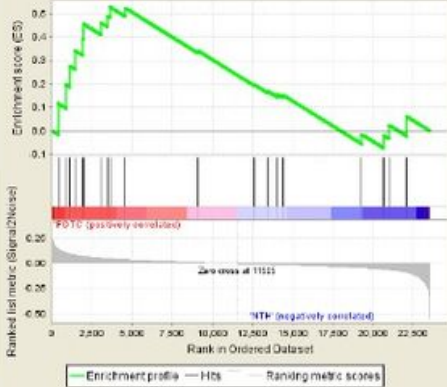

\section{Figure 9}

The enrichment plot of these KEGG pathway in PDTC-NTH. 
A Enrichment plot: KEGG_TRYPTOPHAN_METABOLISM

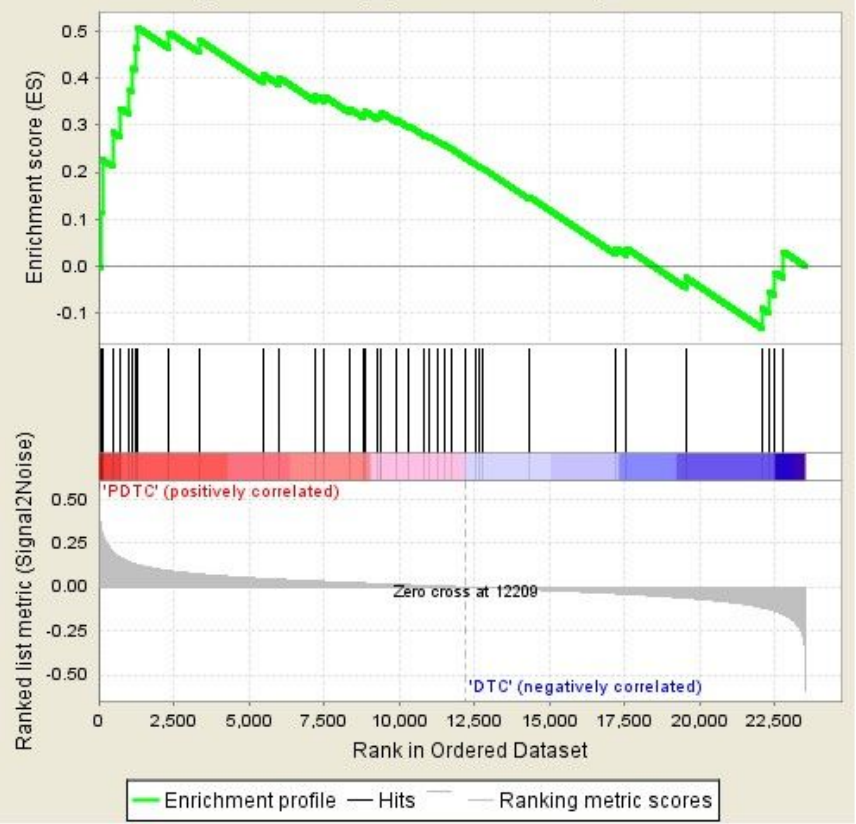

Enrichment plot: KEGG_STARCH_AND_SUCROSE_METABOLISM

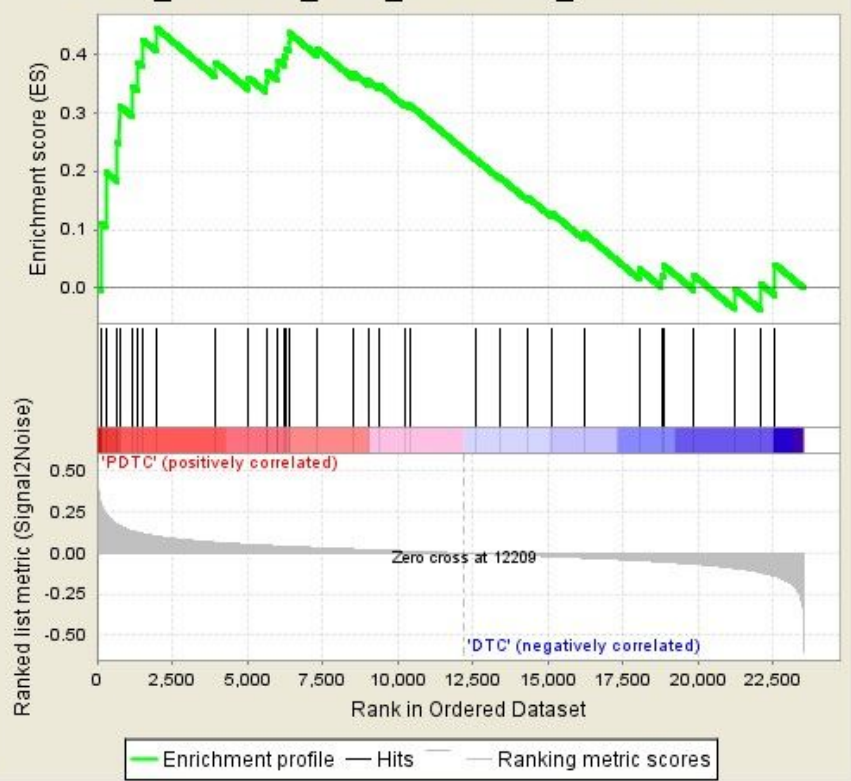

Figure 10

The enrichment plot of these KEGG pathway in PDTC-DTH.

\section{Supplementary Files}

This is a list of supplementary files associated with this preprint. Click to download.

- SupplementaryMaterials.docx 